\title{
Research on the Solar Concentrating Optical System for Solar Energy Utilization
}

\author{
Yimeng Duan, Huajun Yang*, Ping Jiang, and Ping Wang \\ School of physical Electronics, University of Electronic Science and Technology of China, \\ Chengdu 610054, China
}

(Received May 27, 2013 : revised July 8, 2013 : accepted August 6, 2013)

\begin{abstract}
To improve the utilization efficiency of solar energy, a new solar optical concentrating system composed of a parabolic reflector with a square cross-section, a hyperbolic reflector with a square cross-section and two converging convex lenses has been designed. The proposed method can simultaneously focus and shape sun light into a square pattern on the solar panel. In addition, the total reflection property of photonic crystal within the range of the visible sunlight spectrum has been analyzed. Finally, the relationship between solar concentrating multiples and the diameter of the primary mirror has been discussed.
\end{abstract}

Keywords : Parabolic reflector, Hyperboloid reflector, Solar concentrating optical system, The solar concentrating multiples

OCIS codes : (080.2740) Geometric optical design; (080.4035) Mirror system design; (350.6050) Solar energy

\section{INTRODUCTION}

In the past 30 years, the development of new and renewable clean energy has become an issue common to all of the world. In order to improve the utilization efficiency of solar energy, one of the effective ways to reduce the cost of solar power is the use of a solar concentrating system [1-3]. Among the traditional optical shapes, the two stage mirrors reflective configuration has been widely utilized to improve collection efficiency and to decrease the number of expensive cells [4]. The photovoltaic cells are usually available only in rectangular shapes due to fabrication and packaging simplicity [5].

To further enhance the utilization efficiency of the square photovoltaic cell, it is necessary to generate a square light pattern by adopting a parabolic reflector with a square-beam shaping ability [6, 7]. Hence, this paper proposes a new type of solar concentrating optical system. In addition, the total reflection properties of photonic crystal within the range of the visible sun light spectrum has also been analyzed.

\section{DESIGN OF THE SOLAR CONCENTRATING OPTICAL SYSTEM}

The solar concentrating optical system consists of a parabolic reflecting mirror (i.e. the primary mirror) with a square cross-section, hyperbolic reflecting mirror (i.e. the secondary mirror) with a square cross-section, two converging convex lenses and a solar panel. Different from the traditional solar concentrator, this design can shape the sunlight into a square, to just meet the rectangular configuration of solar panels. In order to make more effective use of solar energy, we design a suitable hole in the center of the secondary mirror. By calculating, the secondary mirror size should meet the condition that incident light from the outer edge of the primary mirror just reflects to the outer edge of the secondary mirror, the size of the hole in secondary mirror should meet the condition that the incident light from inner primary mirror edge just reflects to the inner edge of the secondary mirror. The practical structure diagram of the solar concentrating system is shown in Fig. 1.

We place the solar panel at the back of the primary mirror, and put two converging convex lenses in front of the secondary mirror. The two converging convex lenses

\footnotetext{
*Corresponding author: yanghj@uestc.edu.cn

Color versions of one or more of the figures in this paper are available online.
} 


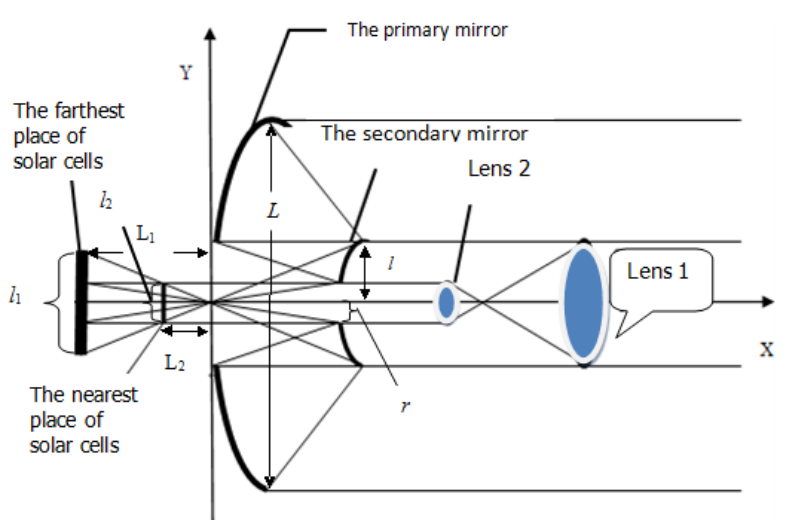

FIG. 1. The solar concentrating system structure.

are used to collect the sunlight blocked by the secondary mirror. As long as the focal point of the lens 1 and the lens 2 is overlapped, the parallel sunlight can also keep parallel after passing through the two lenses. This design makes the center of the solar panel obtain sunlight exposure, and reduces the internal energy consumption caused by uneven distribution of sunlight.

In the meridional plane, the two-dimensional curve equation of the secondary mirror can be described as:

$$
\frac{(x-c)^{2}}{a^{2}}-\frac{y^{2}}{b^{2}}=1
$$

Where $a$ is the real semi-axis of the hyperbola, $b$ is the imaginary semi-axis of hyperbola. $c$ is decided by

$$
c^{2}=a^{2}+b^{2}
$$

The focal point of the two-dimensional secondary mirror is $(0,0),(2 c, 0)$.

In the meridional plane, the two-dimensional curve equation of the primary mirror can be described as:

$$
y^{2}=2 p x
$$

where the $p=4 c$, the focal point of the primary mirror is $(2 c, 0)$. According to the above requirements, the aperture radius $r$ of the secondary mirror is represented as

$$
r=\frac{2 b^{2}\left(\frac{2 l p c-p^{2} l}{l^{2}-p^{2}}\right)+\sqrt{4 b^{4}\left(\frac{2 p c p^{2} l}{l^{2}-p^{2}}\right)^{2}-4\left(b^{2}-\left(\frac{2 l p}{l^{2}-p^{2}}\right) a^{2} b^{2}\left(\left(\frac{2 p c p^{2} l^{2}}{l^{2}-p^{2}}\right)-\left(\frac{2 l p}{l^{2}-p^{2}}\right) a^{2}\right)\right.}}{2\left(b^{2}-\left(\frac{2 l p}{l^{2}-p^{2}}\right) a^{2}\right)}
$$

where $2 l$ is the diameter of the secondary mirror. When we determine the position of the secondary mirror, the $l$ value is determined.

Two-dimensional simulation of the solar concentrating

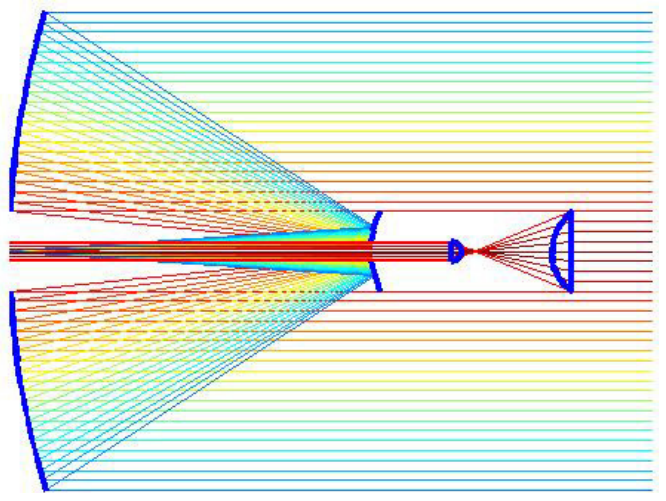

FIG. 2. Two-dimensional simulation of the solar concentrating system.

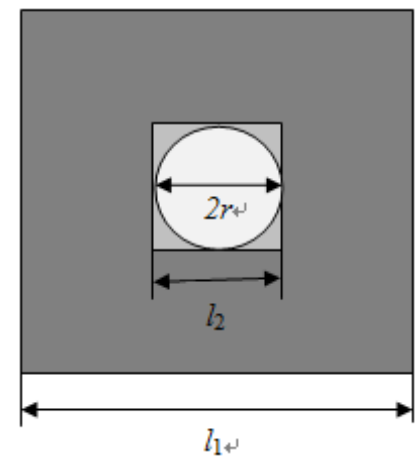

FIG. 3. The light distribution on the solar panel.

system is shown in Fig. 2.

In order to guarantee that all the area of the solar panel is irradiated by sunlight, there should be no gap between the circular spot in the middle and the square spot of the periphery. The sun light which illuminates on the solar panel is shown in Fig. 3.

The bigger rectangle is the light distribution on the solar panel when the solar panel is farthest from the parabolic reflector. The smaller rectangle is the light distribution on the solar panel when the solar panel is nearest from the parabolic reflector.

As is shown in Fig. 3 , the width of the solar panel $l_{1}$ is given by

$$
l_{1}=\frac{2 l\left(\sqrt{1+r^{2} / b^{2}}+\sqrt{1+b^{2} / a^{2}}\right)}{\sqrt{1+l^{2} / b^{2}}+\sqrt{1+b^{2} / a^{2}}}
$$

When the width of the solar panel is maximum, the distance $L_{1}$ between the solar panel and the primary mirror can be described as

$$
L_{1}=a\left(\sqrt{1+r^{2} / b^{2}}+\sqrt{1+b^{2} / a^{2}}\right)
$$


When the solar panel is moved in the direction close to the primary mirror, the solar panel area is also reduced accordingly. The width of the solar panel $l_{2}$ is given by

$$
l_{2}=2 r
$$

When the width of solar panel is minimum, the distance $L_{2}$ between the solar panel and the primary mirror can be described as

$$
L_{2}=\frac{r a\left(\sqrt{1+l^{2} / b^{2}}+\sqrt{1+b^{2} / a^{2}}\right)}{l}
$$

We define the solar concentrating multiples as the ratio of primary mirror area and solar panel area. When the width of the solar panel is $l_{1}$, the solar concentrating multiple is $L^{2} / l_{1}^{2}$. When the width of the solar panel is $l_{2}$, the solar concentrating multiple is $L^{2} / l_{2}^{2}$. The $L$ is the primary mirror diameter, it can be derived as

$$
L=8 c \sqrt{\frac{a^{2}}{b^{2}}+\frac{a^{2}}{l^{2}}}-\frac{8 c^{2}}{l}+8 c \sqrt{\frac{a^{2}}{b^{2}}+\frac{a^{2}}{l^{2}}+\frac{b^{2}}{l^{2}}+1-\frac{2 c}{l^{2}} \sqrt{\frac{l^{2} a^{2}}{b^{2}}+a^{2}}}
$$

To get a concrete relationship between the concentrating multiples and the primary mirror diameter, we should determine the ratio of $a$ and $b$. Assuming that $a: b=4: 3$, the relationship between the solar concentrating multiples and the diameter the primary mirror is shown in Fig. 4.

The primary mirror diameter $L$ value, which can be changed from $0.2 \mathrm{~m}$ to $1.6 \mathrm{~m}$ has been simulated. The situation 1 (solid line) shows that the maximum concentrating multiple is 1148.5 times when the diameter of the primary mirror is minimum (i.e. $0.2 \mathrm{~m}$ ). The situation 2 (dashed line) in Fig. 4 shows that the minimum concentrating multiples of the solar concentrating system is 83.87 times when the diameter of the primary mirror is maximum (i.e.

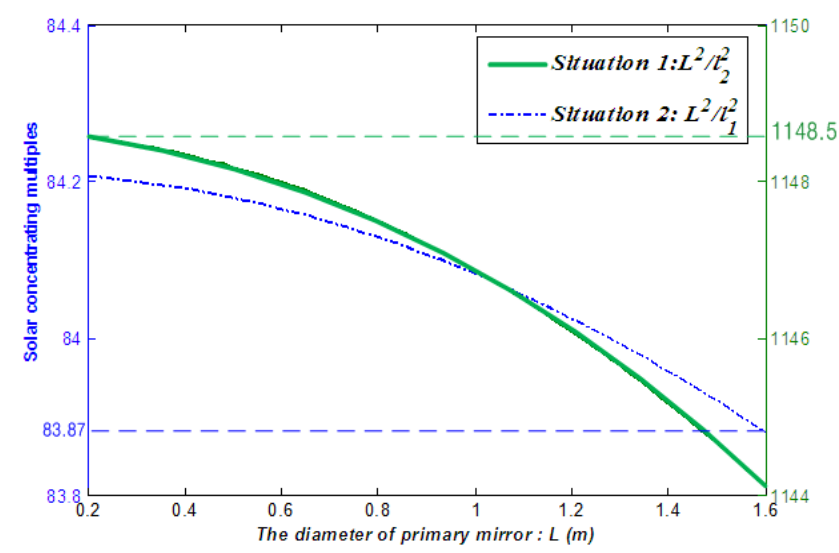

FIG. 4. The relationship between the solar concentrating multiples and the diameter of primary mirror.
$1.6 \mathrm{~m})$. It is also indicated that with the change of primary mirror diameter, the solar concentrating multiples change a little for the same situation.

\section{PROPERTIES OF ONE-DIMENSIONAL PHOTONIC CRYSTAL TOTAL REFLECTION MEMBRANE}

In this paper, the surface for the primary mirror and secondary mirror for the solar concentrating optical system are designed as a one-dimensional photonic crystal structure (the primary mirror and the secondary mirror can be seen in Fig. 1). With this kind of photonic crystal, we can realize a total refection in the sunlight wavelength region.

One-dimensional photonic crystal can be designed as a kind of periodic medium reflector with high reflectivity $[8,9]$. It breaks the limits of metal reflector and periodic medium reflector for realization of low loss and omnidirectional reflection. So it can be applied in many fields such as solar power collection [10,11].

In the TM mode, by using the Maxwell equations, the electric field can be written as [12]

$$
\begin{aligned}
H_{y}^{(j)} & =H^{(j)+} \exp \left(i k_{x} x\right) \exp \left[i k_{z}^{(j)}\left(z-z_{0}^{(j)}\right)\right] \\
& +H^{(j)-} \exp \left(i k_{x} x\right) \exp \left[-i k_{z}^{(j)}\left(z-z_{0}^{(j+1)}\right)\right] \\
E_{x}^{(j)}= & \left(k_{z}^{(j)} / \omega \varepsilon^{(j)}\right) H^{(j)+} \exp \left(i k_{x} x\right) \exp \left[k_{z}^{(j)}\left(z-z_{0}^{(j)}\right)\right]+ \\
& \left(-k_{z}^{(j)} / \omega \varepsilon^{(j)}\right) H^{(j)-} \exp \left(i k_{x} x\right) \exp \left[-i k_{z}^{(j)}\left(z-z_{0}^{(j+1)}\right)\right]
\end{aligned}
$$

where the $j=1,2$, correspond to the two layers of a photonic crystal media material.

The propagation constant of $\mathrm{z}$ direction can be described as

$$
k_{z}^{(j)}=\sqrt{\omega^{2} \varepsilon^{(j)} / c^{2}-k_{x}^{2}}
$$

Where $c$ is the speed of light in vacuum, $\omega$ is the frequency of light, $\varepsilon$ is the permittivity, $k_{x}$ is a constant of electromagnetic waves in the $\mathrm{x}$ direction. Based on the transfer matrix theory and the Bloch theorem [12].

$$
\left(\begin{array}{l}
H^{(2)+} \\
H^{(2)-}
\end{array}\right)=\left(\begin{array}{l}
T_{11} T_{12} \\
T_{21} T_{22}
\end{array}\right)\left(\begin{array}{l}
H^{(1)+} \\
H^{(1)-}
\end{array}\right)=\exp (i K a)\left(\begin{array}{l}
H^{(1)+} \\
H^{(1)-}
\end{array}\right)
$$

The transfer matrix elements can be given

$$
\begin{aligned}
T_{11} & =\left[(1+\Delta)^{2} / 4 \Delta\right] \exp \left[i\left(p_{1}+p_{2}\right)\right] \\
& +\left[-(1-\Delta)^{2} / 4 \Delta\right] \exp \left[-i\left(p_{2}-p_{1}\right)\right]
\end{aligned}
$$




$$
\begin{aligned}
T_{22} & =\left[(1+\Delta)^{2} / 4 \Delta\right] \exp \left[-i\left(p_{1}+p_{2}\right)\right] \\
& +\left[-(1-\Delta)^{2} / 4 \Delta\right] \exp \left[i\left(p_{2}-p_{1}\right)\right] \\
T_{12} & =\left[\left(\Delta^{2}-1\right) / 4 \Delta\right] \exp \left[-i\left(p_{1}+p_{2}\right)\right] \\
+ & {\left[(1-\Delta)^{2} / 4 \Delta\right] \exp \left[i\left(p_{2}-p_{1}\right)\right] } \\
T_{21} & =\left[\left(\Delta^{2}-1\right) / 4 \Delta\right] \exp \left[i\left(p_{1}+p_{2}\right)\right] \\
& +\left[(1-\Delta)^{2} / 4 \Delta\right] \exp \left[-i\left(p_{2}-p_{1}\right)\right]
\end{aligned}
$$

where $K$ is the number of Bloch waves, $a$ is the thickness of a cycle, its range of the first Brillouin zone is $[-\pi / a, \pi / a]$. TM mode eigenvalue equation by the formula

$$
\cos (K a)=\cos p_{1} \cos p_{2}-\frac{1}{2}\left(\Delta+\frac{1}{\Delta}\right) \sin p_{1} \sin p_{2}
$$

Where $p_{j}$ and $\Delta$ are given by the following equations.

$$
\begin{aligned}
& p_{j}=k_{z}^{(j)} h_{j} \\
& \Delta=\varepsilon_{2} k_{z}^{(1)} / \varepsilon_{1} k_{z}^{(2)}
\end{aligned}
$$

By changing the refractive index and the thickness ratio of the photonic crystal, the optimized total reflection band wavelength will be obtained.

In this paper, a period of one-dimensional photonic crystal unit has been designed as two kinds of of medium material. Ratio of the refractive index between the two materials is $n_{1}: n_{2}$ (i.e. the ratio of dielectric constant), the thickness ratio is $h_{1}: h_{2}$.

The part region suface structure of the photonic crystal for the primiary reflection mirror and secondary reflection mirror are shown in Fig. 5. The structure parameters of height, width and period interval can be seen from Fig. 5.

The rectangular part is a total reflection band and the red part is a photonic band gap. We design a rational structure of the photonic crystal, so that the visible sunlight range is included in the total reflection band. This kind of photonic crystal can be a light reflector for its good performance.

When we set $a=0.28 \mu \mathrm{m}$, the photonic band diagrams of three typical structures are shown in Fig. 6.

From Fig. 6, we can get the result that when the photonic crystal medium thickness ratio is fixed, with the increase of the ratio of dielectric refractive index, the total reflection wavelength range increases accordingly. It is indicated that, when the ratio of the refractive index between the two materials is $n_{1}: n_{2}=0.8: 2$, the thickness

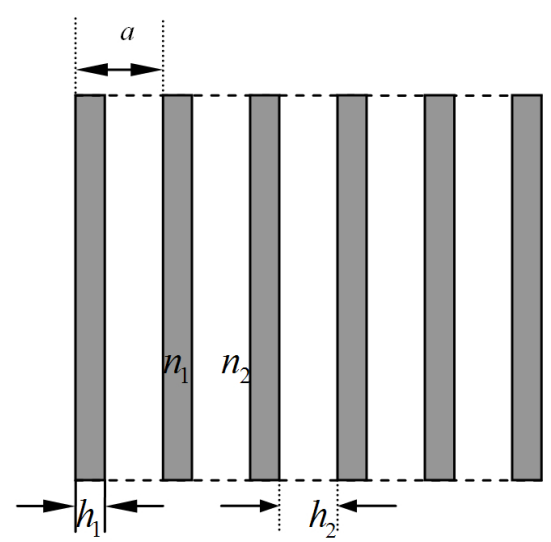

FIG. 5. The structure of the photonic crystal.

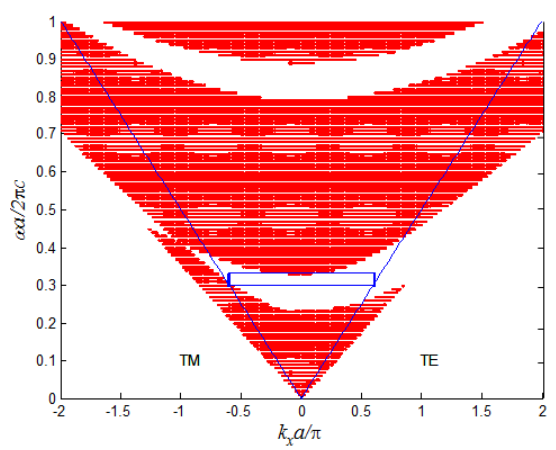

(a)

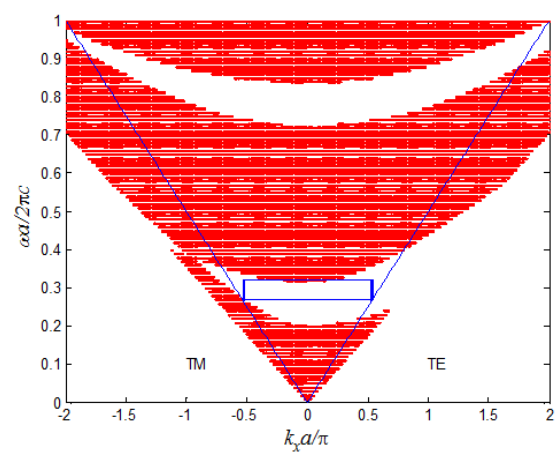

(b)

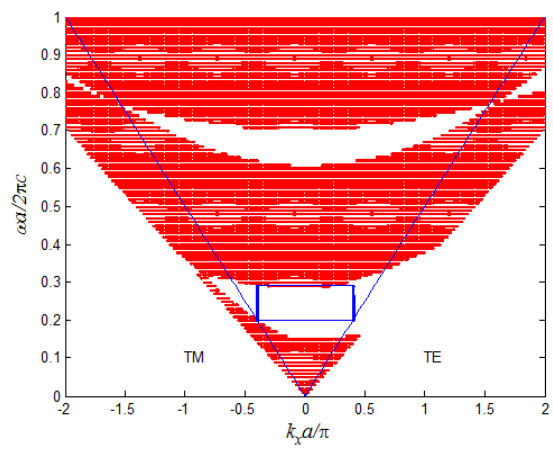

(c)

FIG. 6. The photonic band diagrams of three typical structures. (a) $h_{1}: h_{2}=0.8: 2, n_{1}: n_{2}=4: 1$ (b) $h_{1}: h_{2}=0.8: 2, n_{1}: n_{2}=6: 1$ (c) $h_{1}: h_{2}=0.8: 2, n_{1}: n_{2}=8: 1$ 
ratio is designed as $h_{1}: h_{2}=0.8: 2$, we design the value of $a=0.28 \mu \mathrm{m}$, therefore $h_{1}=0.08 \mu \mathrm{m}, h_{2}=0.20 \mu \mathrm{m}$, the total reflection wavelength range is $380 \mathrm{~nm} \sim 850 \mathrm{~nm}$. This type of photonic crystal mirror can reach a high total reflection in the visible sun light wavelength range.

\section{CONCLUSION}

We have designed a new solar concentrating optical system to shape incident sun rays into a square spot for improving utilization efficiency. The solar concentrating optical system is composed of a parabolic reflecting mirror with a square cross-section, hyperbolic reflecting mirror with a square cross-section, two converging convex lenses and a solar panel. The solar concentrating multiples ranges from 83.874 times to 1148.5 times when the ratio of parameters $a$ and $b$ is $4: 3$, and with the change of primary mirror diameter, the solar concentrating multiple changes a little. In addition, when the ratio of the refractive index between the two material is $n_{1}: n_{2}=0.8: 2$, the thickness ratio is $h_{1}: h_{2}=0.8: 2$, the total reflection wavelength range can be achieved at $380 \mathrm{~nm} \sim 850 \mathrm{~nm}$. This type of photonic crystal mirror can realize a total reflection in the visible sun light wavelength range.

\section{ACKNOWLEDGMENT}

This research is supported by the National Natural Science Foundation of China under Grant No. 61271167.

\section{REFERENCES}

1. S. Kurtz and J. Geisz, "Multijunction solar cells for conversion of conversion of concentrated sunlight to electricity," Opt. Express 18, 73-78 (2010).

2. J. H. Karp, E. J. Tremblay, and J. E. Ford, "Planar microoptic solar concentrator," Opt. Express 18, 1122-1133 (2010).

3. R. Winston, "Principle of solar concentrators of a novel design," Sol. Eng. 16, 89-95 (1974).

4. A. Segal, M. Epstain, and A. Yogev, "Hybrid concentrated photovoltaic and thermal power conversion at different spectral bands," Sol. Eng. 76, 591-601 (2004).

5. S. H. Ma, C. M. Tseng, and Y. P. Lee, "Generation of a uniform-square focal spot by a compound lens for solar concentration applications," Appl. Opt. 52, 3058-3059 (2013).

6. O. Homburg, A. Bayer, T. Mitra, J. Meinschien, and L. Aschke, "Beam shaping of high power diode lasers benefits from asymmetrical refractive micro-lens arrays," Proc. SPIE 7062, 70620T (2008).

7. K. Ryu, J. G. Rhee, K. M. Park, and J. Kim, "Concept and design of modular Fresnel lenses for concentration solar PV system," Sol. Eng. 80, 1580-1587 (2006).

8. Y. Frink, J. N. Winn, and S. Fan, "A dielectric omnidirectional reflector," Science 282, 1679-1682 (1998).

9. B. Mahdieh and G. Nosrat, "Duality of photonic crystal radiative structures and antenna arrays," J. Opt. Soc. Korea 14 438-443 (2010).

10. J. D. Joannopoulos, P. R. Villeneuve, and S. Fan, "Photonic crystals: putting a new twist on light," Nature 386, 143149 (1997).

11. V. Lin, G. N. Lawrence, and J. Buck, "Characterization of excimer lasers for application to lenslet array homogenizers," Appl. Opt. 40, 1931-1941 (2001).

12. X. S. Wen, Photonic, Photonic Crystal Theory and Technology (Science Press, Beijing, China, 2006), pp. 110-112. 